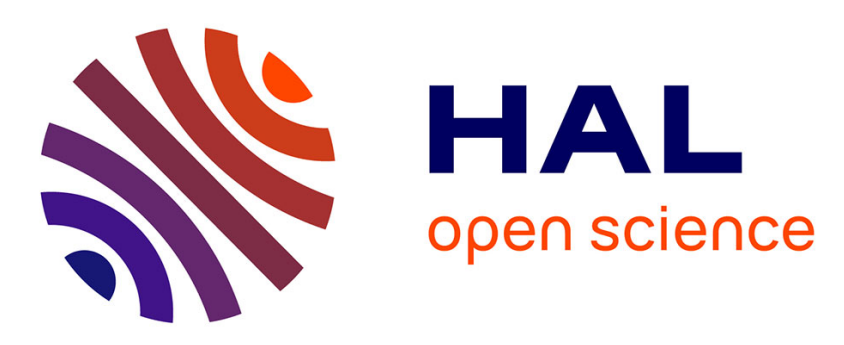

\title{
Recruter les Professeurs d'Université: Le Cas du Concours d'Agrégation du Supérieur en Sciences de Gestion
}

Frédérique Pigeyre, Mareva Sabatier

\section{- To cite this version:}

Frédérique Pigeyre, Mareva Sabatier. Recruter les Professeurs d'Université: Le Cas du Concours d'Agrégation du Supérieur en Sciences de Gestion. Revue française d'administration publique, 2012, 142, pp.399-417. hal-00825990

\section{HAL Id: hal-00825990 https://hal.science/hal-00825990}

Submitted on 25 May 2013

HAL is a multi-disciplinary open access archive for the deposit and dissemination of scientific research documents, whether they are published or not. The documents may come from teaching and research institutions in France or abroad, or from public or private research centers.
L'archive ouverte pluridisciplinaire HAL, est destinée au dépôt et à la diffusion de documents scientifiques de niveau recherche, publiés ou non, émanant des établissements d'enseignement et de recherche français ou étrangers, des laboratoires publics ou privés. 
Frédérique PIGEYRE, Université Paris-Est Créteil, IRG

Mareva SABATIER, Institut de Management de l'Université de Savoie, IREGE

Adresse de correspondance :

Frédérique Pigeyre

IRG - IAE Gustave Eiffel

Université Paris-Est Créteil

Place de la Porte des Champs

Route de Choisy

94010 Créteil Cedex

pigeyre@u-pec.fr

\section{«Recruter les Professeurs d'Université : Le Cas du Concours d'Agrégation du Supérieur en Sciences de Gestion»}

\section{Résumé :}

Ce texte interroge les vertus et les limites des concours nationaux de recrutement dans la Haute Fonction Publique, valorisés comme garantissant à la fois l'intérêt général, en sélectionnant les plus qualifiés, et l'intérêt particulier, en assurant un jugement sur le mérite. Nous étudions plus précisément le dispositif de recrutement des professeurs d'université. Historiquement issues du droit, l'économie, la science politique et les sciences de gestion sont des disciplines dotées d'un concours national d'agrégation du supérieur. La recherche menée porte sur les concours organisés en sciences de gestion, depuis leur création en 1976 jusqu'au concours de 2007.

Nous identifions les facteurs déterminants de la réussite à ces concours. En réalisant des analyses économétriques qui donnent des effets toutes choses égales par ailleurs, nous mettons en évidence un impact significatif de la production scientifique, mais aussi un effet du réseau, quantitativement plus important que celui des publications. Ces résultats interrogent donc le caractère plus ou moins équitable de ces concours.

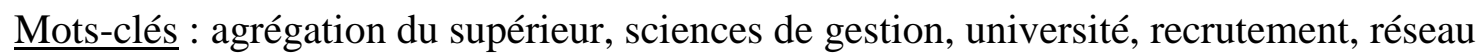




\section{Introduction}

Cet article s'intéresse aux modalités d'évolution des carrières dans la Haute Fonction Publique d'Etat, et plus particulièrement dans le système universitaire en France. A l'heure où ce système connaît d'importants changements (notamment du fait de la loi LRU d'août 2007), il semble particulièrement opportun de s'interroger sur les avantages et les limites des systèmes en vigueur.

La France se caractérise en effet par un système de Fonction Publique dit « de carrière », par opposition aux systèmes dits «d'emploi » des pays nordiques par exemple, qui privilégie le concours comme dispositif de recrutement quasi-exclusif des agents de l'Etat. Le concours est supposé garantir simultanément l'intérêt général, en sélectionnant les plus qualifiés, et l'intérêt particulier, les candidats étant assurés d'être jugés selon leurs mérites ${ }^{1}$. Avant la généralisation du concours, le recrutement dans la Fonction Publique est resté durablement marqué par le népotisme et le clientélisme ${ }^{2}$.

Le concours national sur épreuves anonymes qui permet de sélectionner les meilleurs parmi un nombre important de candidats constitue ainsi le modèle emblématique du concours. Force est de constater que la réalité est bien différente. En réalité de nombreuses modalités de concours existent, variables selon les Ministères et selon les postes à pourvoir, qui s'éloignent peu ou prou de ce modèle.

L'Université française, institution étatique, n'échappe pas à cette réalité. Elle recrute ses enseignants-chercheurs ${ }^{3}$ titulaires par le biais de concours. Cette situation diffère de celle de la plupart des pays industrialisés qui ont essentiellement recours au modèle du marché 4 . Toutefois, malgré les différences importantes entre ces deux formes de recrutement, elles prétendent autant l'une que l'autre répondre à la conception universaliste de la profession académique décrite par une abondante littérature, notamment anglo-saxonne. Cette conception universaliste de la science, qui valorise exclusivement la productivité scientifique (publications et reconnaissance par les pairs, notamment), détermine une vision normative du recrutement des membres de la profession, uniquement fondé sur le jugement équitable des seules qualités scientifiques. De nombreux auteurs nord-américains ${ }^{5}$ ont néanmoins montré que cet idéal mertonien, constitutif d'une éthique scientifique universelle, n'était pourtant pas toujours respecté. De nombreuses institutions universitaires doivent ainsi composer avec d'autres exigences que la seule productivité scientifique, les conduisant à privilégier chez les personnes recrutées d'autres qualités telles que les compétences pédagogiques, l'investissement au service de l'institution, la capacité à développer de nouveaux programmes, etc.

\footnotetext{
${ }^{1}$ Sadran (Pierre), «Recrutement et sélection par concours dans l'administration française », Revue Française d'administration publique, $\mathrm{n}^{\circ}$ 1, 1977, p. 53-107.

${ }^{2}$ Holcman (Robert), «Secteur public, secteur privé : similarités et différences dans la gestion des ressources humaines », Revue française d'administration publique, Vol. 123 (3), 2007, p. 409-421

${ }^{3}$ Nous nous intéressons ici à la seule profession académique, et non pas aux personnels Biatoss qui relèvent d'une autre forme de concours et de recrutement.

${ }^{4}$ Musselin (Christine), Le marché des universitaires, Paris, Presses de la Fondation nationale de sciences politiques, 2005 ;

5 Crane (Diana), «The academic marketplace revisited », American Journal of Sociology, Vol. 75 (6), 1970, p. 953-964; Long (J. Scott), Allison (Paul D.) et McGinnis (Robert), « Rank Advancement in Academic Careers: Sex Differences and the Effects of Productivity », American Sociological Review, Vol. 58 (5), 1993, p.703-722; Long (J. Scott) et Fox (Mary F.), «Scientific careers : universalism and particularism », Annual Review of Sociology, Vol. 21, 1995, p. 45-71.
} 
On pourrait donc se poser la question de savoir lequel, du «modèle du marché » ou du «modèle du concours », correspond le mieux à cet idéal universel. Mais on peut tout autant s'interroger sur l'intérêt qu'il y a à privilégier exclusivement un tel idéal. D'un strict point de vue de l'efficacité, il convient en effet de se demander si le meilleur candidat dans une université donnée à un emploi donné de professeur est forcément celui qui répond parfaitement aux seuls critères de productivité scientifique. En outre, une analyse comparée de ces deux types de systèmes de recrutement montre qu'aucun n'est exempt de biais et que ceux-ci sont différents d'un modèle à l'autre, notamment quant aux inégalités qu'ils créent ${ }^{6}$.

L'université française n'échappe donc pas à ces débats sur les avantages et les limites comparés des «concours » - même s'ils s'avèrent, de fait, assez éloignés du modèle emblématique précité -, et des «recrutements », au sens d'appariement entre un profil de poste donné et un nombre fini de candidats, parfois modeste, parmi lesquels il s'agit de choisir le meilleur.

Une analyse précise des modalités de recrutement des professeurs apparait particulièrement nécessaire pour alimenter ce débat, au moins pour deux raisons.

La première raison tient au fait que l'on observe que les modalités de ces concours de recrutement ne sont pas équivalentes dans toutes les disciplines. Quatre d'entre elles, le droit (qui inclus droit privé, droit public et histoire du droit), l'économie, la science politique et les sciences de gestion, disposent de concours nationaux d'agrégation du supérieur, un concours dit externe et l'autre dit «interne» organisés alternativement une année sur deux, les concours externes fournissant le très grande majorité des professeurs. Dans toutes les autres disciplines, à savoir près de 70 d'entre elles, les professeurs sont recrutés par des comités de sélection mis en place au sein des universités ${ }^{7}$. La seconde raison est que l'accès au grade de professeur représente un enjeu majeur pour la profession universitaire et pour ses membres. Il en constitue en effet l'étape ultime de carrière qui apparaît d'ailleurs comme assez difficilement accessible si l'on considère la part relativement faible du nombre des professeurs dans l'ensemble de la profession (34\% en 2007, toutes disciplines confondues).

Notre analyse porte sur les sciences de gestion, que nous avons étudiées dans le cadre d'un projet de recherche plus large sur les carrières des universitaires, qui associe à la fois des approches quantitatives (analyses statistiques et économétriques à partir des données du Ministère de l'enseignement supérieur et de la recherche) et qualitatives (entretiens biographiques approfondis auprès d'universitaires). Dans cette discipline, environ $70 \%$ des professeurs le sont devenus via les concours d'agrégation et parmi eux, 85\% par le concours externe. L'agrégation, qu'elle soit interne ou externe ${ }^{8}$, apparaît comme une épreuve à la fois difficile et sélective nécessitant une importante préparation. Parée de nombreuses vertus pour les uns, qui voient en elle la garantie d'une sélection scientifique rigoureuse des candidats et d'une protection contre un «localisme ${ }^{9}$ dépassé, elle est fortement critiquée par les autres qui la considèrent plutôt comme une forme excessive de cooptation par les pairs.

Pour tenter d'y voir plus clair, nous avons procédé à une analyse des déterminants de la réussite au concours externe d'agrégation. Une telle analyse nous permet en effet de mettre à

\footnotetext{
${ }^{6}$ Musselin (Christine), Pigeyre (Frédérique) et Sabatier (Mareva), « National competition versus recruitment processes : which inequalities do they favour? », Communication au colloque du RESUP, Lausanne, 2009

${ }^{7}$ Il s'agit principalement de commissions de spécialistes, constituées au sein des établissements recruteurs, de professeurs qui choisissent leur(s) futur(s) pair(s) sur la base d'analyse, en termes d'activités pédagogiques et de recherche, du dossier de candidature et d'une audition.

${ }^{8}$ Voir infra.

9 Voir le débat sur le localisme Godechot (Olivier) et Louvet (Alexandra), «Le localisme dans le monde universitaire : un essai d'évaluation », La Vie des Idées, 2008. http://www.laviedesidees.fr/IMG/pdf/20080422_localisme.pdf
} 
jour les critères effectivement retenus dans les concours, et de vérifier ainsi en quoi il s'approche ou s'éloigne de l'idéal mertonien. Plus largement, nous tentons d'évaluer si le concours d'agrégation permet de garantir à la fois l'intérêt général et celui des candidats et donc de caractériser ses principales vertus.

Après avoir rappelé dans un premier temps les caractéristiques du concours externe d'agrégation et évoqué les résultats de certaines études disponibles sur le sujet, nous expliciterons notre méthodologie de recherche, puis les principaux résultats obtenus. Nous conclurons sur les évolutions qu'il est possible d'envisager, dans un contexte aujourd'hui marqué par la concurrence accrue des grandes écoles, le départ à la retraite de nombreux professeurs dans les cinq années à venir et la pénurie annoncée de docteurs en gestion.

\section{L'accès au grade de Professeur}

\subsection{Historique et organisation du concours d'agrégation externe}

Issues des sciences économiques, les sciences de gestion en ont adopté les structures : elles se sont dotées d'un concours national d'agrégation du supérieur pour choisir leurs professeurs. Deux concours existent : l'un dit externe, auquel peuvent postuler tous les titulaires d'un doctorat et l'autre dit interne, réservé aux maîtres de conférences ayant 40 ans révolus au $1^{\text {er }}$ janvier de l'année du concours. Nous focalisons ici l'attention sur le seul concours externe, car seulement quatre concours internes ont eu lieu pour un nombre limité d'agrégés par cette voie (47). En revanche, dix-sept concours externes ont été organisés de 1976 à 2009 et ont permis de recruter 307 Professeurs des Universités.

Les concours d'agrégation constituent des procédures longues qui suivent différentes étapes. Dans un premier temps sont publiés au Journal Officiel l'annonce du concours, le nombre de postes ouverts et la date limite de candidature. Le plus souvent, avant cette date limite, le Ministère nomme le Président du jury en fonction de son ancienneté dans le grade de professeur et selon un équilibre Paris/Province. Le Président choisit à son tour les autres membres, parmi lesquels une ou deux personnalités représentant le monde économique. Chaque concours est donc unique, le jury étant composé de façon ad hoc.

Ensuite, les épreuves se déroulent généralement de septembre à mai. A partir de 1984 et jusqu'en 2000, le concours comportait quatre épreuves successives, constituant pour les candidats autant d'étapes à franchir ${ }^{10}$. La première épreuve consiste en un exposé des travaux de recherche du candidat à la suite duquel il est interrogé sur différents points relatifs à ses travaux. La seconde épreuve, la leçon de huit heures en loge, permet de tester la culture générale en gestion des candidats. La troisième épreuve constitue la leçon de spécialité ${ }^{11}$. L'épreuve de 24 heures, supprimée en 2000, nécessitait la constitution d'une équipe pluridisciplinaire permettant de réaliser une étude de cas.

Après la dernière épreuve, le jury prononce les noms des lauréats et donne leur classement. La localisation des postes est alors rendue publique et les nouveaux agrégés, par ordre de classement, choisissent leur établissement d'affectation.

\footnotetext{
${ }^{10}$ Désormais, les candidats peuvent être éliminés à l'issue de chaque épreuve. Lorsqu'il existait quatre épreuves, une sous-admissibilité était prononcée à l'issue de la première épreuve puis l'admissibilité après la seconde épreuve et les candidats restant passaient la troisième et la quatrième épreuve.

${ }^{11}$ Huit spécialités ont été définies pour le dernier concours : comptabilité et contrôle, finance, marketing, gestion des ressources humaines, gestion de production et logistique, gestion des systèmes d'informations et communication, gestion juridique et fiscale, management stratégique.
} 
Au-delà de ces éléments descriptifs, il convient d'étudier les facteurs de réussite à ce concours.

\subsection{Quelques éléments comparatifs}

La grande majorité des études sur les carrières des universitaires et notamment sur l'accès au grade de professeur concernent les pays anglo-saxons, et notamment les Etats-Unis. Elles portent sur les promotions du grade d'Assistant Professor (équivalent au statut de maître de conférences) à celui de Full Professor (équivalent des Professeurs des Universités) aux EtatsUnis. Bien qu'elles s'intéressent à un tout autre contexte institutionnel, elles fournissent des pistes intéressantes sur les déterminants de l'accès au grade universitaire le plus élevé dans les universités.

Parmi les facteurs explicatifs les plus importants mis en exergue, figure le genre : toutes ces études rendent compte de l'existence d'un plafond de verre à l'Université. Même si leur part augmente parmi les universitaires, les femmes peinent à atteindre les postes les plus élevés dans la hiérarchie. Elles ont ainsi, toutes choses égales par ailleurs, c'est-à-dire en les comparant avec des hommes qui auraient des caractéristiques équivalentes par ailleurs (même âge, même université de rattachement, même dossier scientifique...), une probabilité plus faible de passer au grade de Full Professor, quelle que soit la discipline.

Par exemple en sciences humaines, les femmes ont une probabilité de devenir Full Professor de $8 \%$ inférieure à celles des hommes, toutes choses égales par ailleurs ${ }^{12}$. En sciences économiques, le différentiel serait de 7,6 points en défaveur des femmes dans les universités du Sud des Etats-Unis, après prise en compte des potentielles différences sexuées en termes de publications ${ }^{13}$. Des résultats similaires pour le système universitaire britannique ont également pu être mis en évidence ${ }^{14}$.

Au delà de l'effet du genre, les études anglo-saxonnes rendent compte de l'impact important de la production scientifique. En sciences, les Assistant Professors les plus publiants ont ainsi de plus fortes chances d'être promus au grade supérieur ${ }^{15}$. Non seulement le nombre de publications agit comme un accélérateur de promotions, mais la qualité de ces dernières joue aussi un rôle moteur.

Mais ces études portent sur des systèmes universitaires bien différents du système français, caractérisés notamment par une très nette décentralisation des prises de décision et de gestion des carrières des universitaires. En France, très peu d'analyses ont été entreprises pour étudier les déterminants de l'accès au grade de Professeur des Universités. La première étude descriptive du concours d'agrégation en sciences économiques a été produite en $2004^{16}$. Elle met en avant l'absence d'un effet de genre mais une forte prime à la jeunesse et un effet important de la proximité avec un membre du jury. Ces résultats ont d'ailleurs été confirmés ${ }^{17}$

\footnotetext{
12 Ginther (Donna K.) et Hayes (Kathy J.), «Gender Differences in Salary and Promotion for Faculty in the Humanities 1977-1995 », Journal of Human Resources, Vol.38 (1), 2003, p. 34-73.

13 Mixon (Franklin G.) et Trevino (Len J.), «Is there gender discrimination in named professorships? An econometric analysis of economics departments in the US South » Applied Economics, $n^{\circ}$ 37, 2005, p. 849-854.

${ }^{14}$ Ward (Melanie), «Gender and Promotion in Academic Profession », Scottish Journal of Political Economy, Vol. 48 (3), 2001, p. 283-302.

${ }_{16}^{15}$ Long (J. Scott), Allison (Paul D.) et McGinnis (Robert), art. cit.

${ }^{16}$ Linnemer (Laurent) et Perrot (Anne), «Tout ce que vous avez toujours voulu savoir sur le concours d'agrégation en sciences économiques », Revиe Economique, Vol. 55 (2), 2004, p. 275-321.

${ }^{17}$ Combes (Pierre-Philippe), Linnemer (Laurent) et Visser (Michael), « Publish or peer-rich ? The role of skills and networks in hiring economics professors », Labour Economics, Vol. 15 (3), p. 723-742.
} 
par l'estimation économétrique des déterminants de la réussite au concours d'agrégation en économie (1983-2003), en tenant compte du rang de classement. L'étude souligne notamment que les liens avec les membres du jury jouent un rôle central dans la réussite. Ainsi, toutes choses égales par ailleurs, la probabilité de succès est doublée lorsque le candidat a son directeur de thèse dans le jury et même triplée si le candidat a dans son jury, non seulement son directeur de thèse, mais aussi un co-auteur. Cet effet de réseau est même bien plus important dans leur réussite au concours que les publications des chercheurs, qui augmentent pourtant la probabilité de réussite. Pour compenser l'absence du directeur de thèse dans le jury et assurer la même probabilité de réussite, il faudrait avoir publié cinq articles de plus ${ }^{18}$.

Concernant le concours en sciences de gestion une seule étude, centrée sur l'histoire du concours dans cette discipline, a été publiée ${ }^{19}$. Rappelant la genèse du concours, elle présente des statistiques descriptives sur les concours entre 1976 et 2005 qui laissent à penser que le réseau joue un rôle dans la réussite au concours en sciences de gestion, comme en sciences économiques. Mais la base de données exploitée par l'auteur ne renseigne pas sur les publications des candidats, ce qui ne permet pas de mener une analyse plus poussée des déterminants de la réussite au concours.

\section{Eléments de méthodologie}

Pour compléter les analyses disponibles, nous nous sommes essentiellement appuyées sur une approche quantitative. Cela nous permet d'avoir une mesure des facteurs objectifs et quantifiables expliquant la réussite tels que les attributs individuels la production scientifique ou le réseau. Bien entendu, d'autres facteurs, tels le contexte professionnel dans lequel évoluent les enseignants-chercheurs (qualité des laboratoires, tâches administratives...) ou l'environnement personnel (articulation vie professionnelle et vie personnelle par exemple), mériteraient d'être pris en compte. Pourtant, ces facteurs sont extrêmement difficiles à observer, notamment en dynamique. Compte tenu de ces variables manquantes (que seules des analyses qualitatives pourraient permettre d'étudier), la qualité des estimations économétriques (présentées infra) sera systématiquement étudiée afin de mesurer la part nonexpliquée de la probabilité de réussir le concours d'agrégation.

\subsection{Construction de la base de données sur les individus}

Pour étudier de manière fine les déterminants de la réussite, nous avons construit une base de données inédite qui recense pour l'ensemble des candidats aux concours d'agrégation du supérieur en sciences de gestion (1976-2007) ${ }^{20}$ des informations sur leur réussite au concours, le contexte du concours, leurs attributs de carrières, leurs publications ainsi que des variables de réseau.

En l'absence d'une base de données déjà constituée sur le thème, ces informations ont dû être recherchées à partir de différentes sources puisqu'aucune base complète n'existait jusqu'alors. Les données sur les concours (date, noms des candidats et des membres du jury, nombre de postes mis au concours) ont été fournis par le Ministère de l'Enseignement Supérieur et de la Recherche. La base de données SUDOC (Système Universitaire de Documentation) nous a

\footnotetext{
${ }^{18}$ Selon les mêmes auteurs.

${ }^{19}$ Marco (Luc), «L'agrégation en sciences de gestion (1976-2005) », Revue d'histoire des sciences humaines, $\mathrm{n}^{\circ}$ 14, 2006, p. 173-198.

${ }^{20}$ Les deux derniers concours, en 2008-2009 et 2010-2011, n'ont pas pu être pris en compte dans nos analyses.
} 
renseigné des caractéristiques de la thèse (date et université de soutenance, nom du directeur de thèse et spécialité de la thèse).

\subsection{Constitution d'une base de données sur les publications et le réseau}

Un important travail de recherche sur les publications a également été entrepris. Pour chaque enseignant-chercheur, nous avons recensé sa production scientifique réalisée avant le concours. Différentes options s'offraient à nous, notamment l'utilisation de l'indice $h$ (Hirsch, 2005), qui recense à partir de Google Scholar toute la production scientifique en la pondération par les citations de chaque publication. Cet indice, de plus en plus utilisé en bibliométrie, a pour avantage de fournir un indicateur unique de production scientifique mais, par définition, ne permet pas de tenir compte de l'hétérogénéité des travaux (articles, livres...). Pour dépasser ce défaut, nous avons donc opté pour un recensement quantitatif (nombre de publications) et nous avons subdivisé les publications selon leur type: les publications dans des revues anglophones, celles dans les revues francophones et enfin les livres publiés. Les publications anglophones ont été collectées via une recherche nominative sur les bases bibliographiques en ligne (Business Source Premier, Wiley, Econlit, SpringerLink et Science Direct). Pour les publications francophones, les ressources électroniques (telles CAIRN et Vente et Gestion) n'ont pas été suffisantes pour permettre d'avoir une bonne mesure de cette production, certaines revues pourtant reconnues en gestion n'étant pas encore en ligne, ou uniquement pour les numéros les plus récents. Nous avons donc collecté (à la Bibliothèque Nationale) tous les sommaires d'une sélection de revues francophones, sommaires dans lesquels ont été recherchées les contributions des candidats à l'agrégation externe. Enfin, nous avons aussi recensé le nombre de livres écrits par les candidats à partir du fichier de la Bibliothèque Nationale et de la base SUDOC.

Contrairement à certaines études ${ }^{21}$, nous n'avons pas introduit explicitement de critère de qualité dans cette production scientifique. Bien que toutes les publications ne se valent pas, les revues étant de qualité différente, les classements des revues (comme les classements des revues de la section 37 du CNRS, de l'Ecole de Management de Lyon, de l'ESSEC ou de l'AERES) sont assez récents et ne concernent donc que les observations les plus récentes. Ces classements sont en outre souvent différents selon les institutions qui les réalisent si bien qu'un consensus semble ne se dégager que sur un nombre limité de revues. La prise en compte des classements de revues tels qu'ils existent aurait donc contribué à un biais important pour les candidats des premiers concours, quand le classement des revues n'était pas établi, ni même reconnu.

Compte tenu des choix opérés pour établir nos indicateurs de production scientifique, nous avons voulu tester la robustesse de nos mesures et les avons comparées avec le facteur $h$ associé à chaque candidat, à la date du concours ${ }^{22}$. Cette analyse de sensibilité permet d'établir que nos indicateurs sont très fortement corrélés avec l'indice $h$. Le périmètre choisi pour les revues ne semble donc pas particulièrement affecter la mesure de la production, tout en la rendant plus lisible ${ }^{23}$.

\footnotetext{
${ }^{21}$ Notamment celle de Combes et al., art.cit.

${ }^{22}$ Hirsch (Jorge E.), «An index to quantify an individual's scientific research output », arXiv:physics/0508025 Vol.5 (29), 2005. L'indice $h$ peut être obtenu via l'interface suivante : http://www.harzing.com/pop.htm

${ }^{23}$ Notons aussi que les résultats économétriques donnent des effets d'ampleur équivalente si on utile l'indice $h$ plutôt que nos indicateurs de publications et ce, tant au niveau de l'effet de la production scientifique que de l'effet du réseau. Les résultats de cette analyse de sensibilité sont disponibles sur demande auprès des auteurs.
} 
Au delà des variables décrivant la production scientifique des candidats à ce concours, nous avons aussi voulu mesurer le lien éventuel existant entre les membres du jury et les candidats. La littérature a en effet souligné les impacts importants du réseau dans l'accès au grade de Professeur des Universités, via l'agrégation. Deux types de liens ont été définis dans la base de données : avoir un coauteur dans le jury (à partir des informations sur le jury et sur les publications) et avoir son directeur de thèse dans le jury (à partir des informations sur le jury et sur les thèses dans la base SUDOC).

Nous avons aussi construit une variable binaire égale à 1 si le directeur de thèse du candidat a participé à un autre jury d'agrégation que le sien. En distinguant ainsi la participation dans un jury à la participation dans le jury du candidat, il est possible de tester deux effets concomitants de la présence du directeur de thèse : un effet de soutien direct mais aussi un effet de réputation, les directeurs de thèse les plus réputés (et ayant davantage de chances d'être membres de jury d'agrégation) attirant les meilleurs étudiants, la présence du directeur dans un jury s'interprétant alors comme un signal de compétence du candidat.

L'annexe 1 présente la liste des variables disponibles dans la base de données, la source de ces données et quelques statistiques descriptives.

Nous constatons que si certaines informations sont disponibles pour l'ensemble des 626 candidats, d'autres, en raison d'observations manquantes, ne sont pas disponibles pour tous. Ceci concerne surtout les variables créées à partir des données sur les thèses. Notre analyse statistique ne portera donc pas sur l'intégralité des candidats mais uniquement sur ceux dont on dispose de toutes les caractéristiques, soit un échantillon cylindré. Pour ne pas introduire de biais lié au cylindrage, nous intégrons, dans les estimations du taux de réussite à l'agrégation présentées dans la section suivante, une pondération des observations de manière à redresser l'échantillon cylindré afin qu'il présente des caractéristiques moyennes équivalentes à la population de départ.

\section{Les déterminants de la réussite au concours d'agrégation}

Nous présentons dans ce paragraphe les résultats de l'analyse descriptive. Elle permet d'identifier quatre éléments principaux qui caractérisent les lauréats aux différents concours.

\subsection{Des taux de réussite irréguliers selon les périodes}

La base de données construite permet de retracer le parcours des candidats aux quinze concours d'agrégation ainsi que leur réussite ou non à ce concours. En moyenne, le taux de réussite est de 44,7\%, mais il varie fortement selon les concours. Le graphique 1 souligne ainsi que le taux de réussite a oscillé entre 31\% lors du concours de 2006 et $75 \%$ en 1998 et indique une tendance à la baisse du taux de réussite sur les deux derniers concours, qui ont été les plus sélectifs de tous. A l'état brut, ces résultats reflètent principalement le caractère unique de chaque jury. Des analyses qualitatives seraient nécessaires pour procéder à des interprétations fines. 
Graphique 1: Taux de réussite par concours

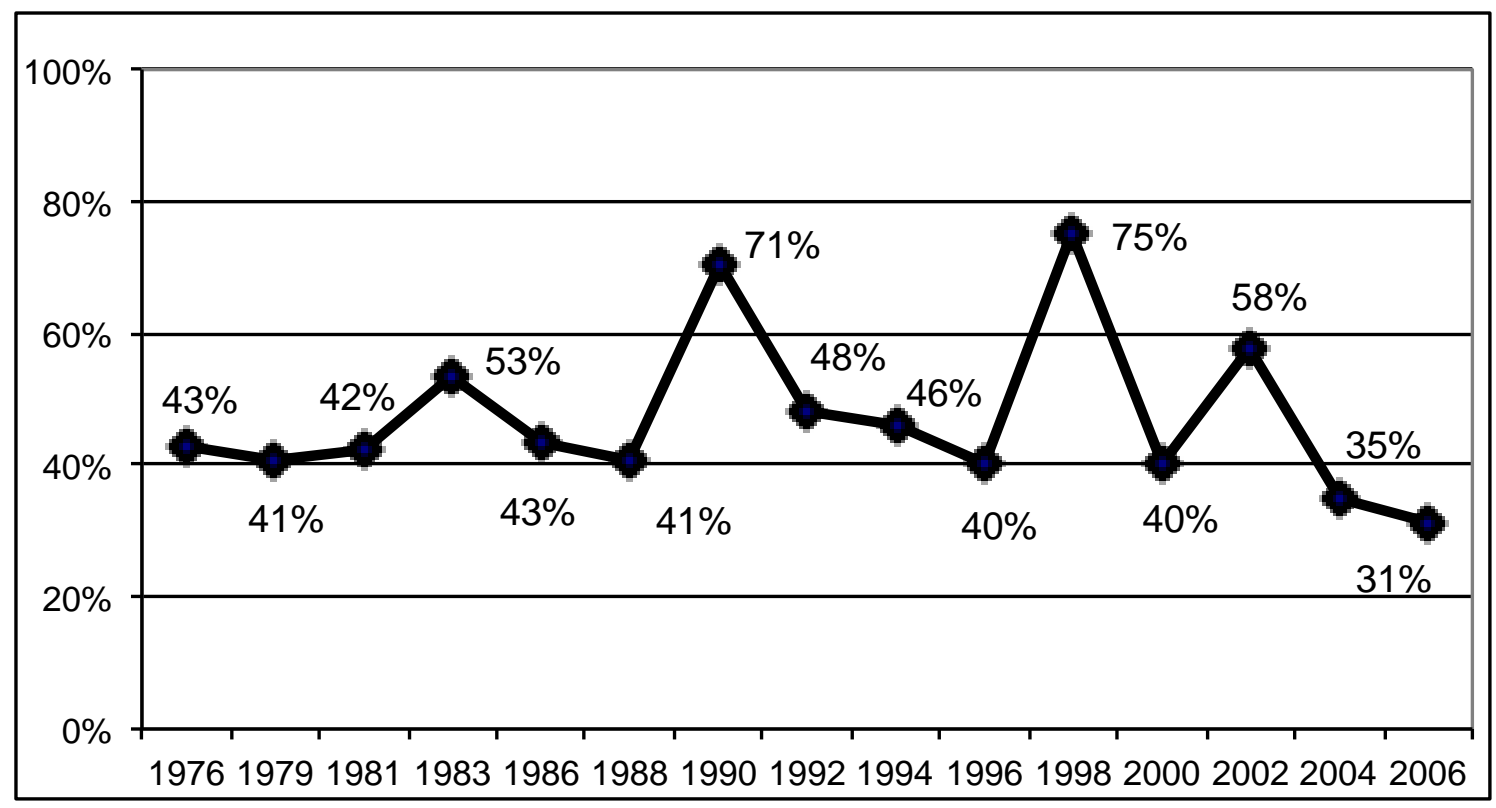

\subsection{Une prime à la jeunesse}

Le tableau 1 précise davantage le profil des candidats réussissant le concours. Parmi les variables qui semblent avoir le plus d'impact, on note une certaine prime à la jeunesse puisque les candidats qui soutiennent leur thèse quand ils sont jeunes et qui mettent le moins de temps à passer le concours (depuis la thèse) sont sur-représentés parmi les lauréats.

$\underline{\text { Tableau } 1: \text { Caractéristiques des agrégés et non-agrégés }}$

\begin{tabular}{|c|c|c|c|}
\hline & Non agrégés & Agrégés & Total \\
\hline fem : être de sexe féminin & $22,0 \%$ & $19,8 \%$ & $20,9 \%$ \\
\hline these_concours : temps entre la thèse et le concours & 7,41 & 4,85 & 6,18 \\
\hline age_these : age lors de la thèse & 31,81 & 30,79 & 31,32 \\
\hline nb_tent : nombre de tentative & 1,49 & 1,30 & 1,40 \\
\hline poste_candidat: degré de concurrence & 0,39 & 0,44 & 0,41 \\
\hline compta fi : avoir une thèse en comptabilité ou finance & $23,5 \%$ & $28,3 \%$ & $25,8 \%$ \\
\hline marketing : avoir une thèse en marketing & $8,0 \%$ & $13,8 \%$ & $10,8 \%$ \\
\hline grh : avoir une thèse en GRH & $8,3 \%$ & $9,7 \%$ & $9,0 \%$ \\
\hline logistiq: avoir une thèse en logistique & $3,8 \%$ & $3,6 \%$ & $3,7 \%$ \\
\hline si : avoir une thèse en comptabilité ou finance & $2,3 \%$ & $3,2 \%$ & $2,7 \%$ \\
\hline strat : avoir une thèse en management stratégique & $23,1 \%$ & $24,3 \%$ & $23,7 \%$ \\
\hline autre_disc: avoir une thèse en dehors des sciences de gestion & $31,1 \%$ & $17,0 \%$ & $24,3 \%$ \\
\hline mcf : être maître de conférence au moment du concours & $73,5 \%$ & $63,2 \%$ & $68,5 \%$ \\
\hline pub_angl : nombre de publications dans des revues anglophones & 0,34 & 0,70 & 0,52 \\
\hline pub_fr : nombre de publications dans des revues francophones & 2,06 & 2,73 & 2,38 \\
\hline livre : nombre de livres publiés & 1,07 & 1,02 & 1,05 \\
\hline directeur_jury : avoir son directeur de thèse dans le jury & $2,3 \%$ & $12,1 \%$ & $7,0 \%$ \\
\hline $\begin{array}{l}\text { direct_autre jury : avoir son directeur de thèse membre d'un autre } \\
\text { jury }\end{array}$ & $23,5 \%$ & $33,2 \%$ & $28,2 \%$ \\
\hline coauteur_jury : avoir un coauteur dans un jury & $1,5 \%$ & $6,5 \%$ & $3,9 \%$ \\
\hline
\end{tabular}

NB : statistiques données pour le sous-échantillon cylindré 
Les résultats descriptifs indiquent par ailleurs que le genre, le nombre de tentatives et la spécialité de la thèse ne semblent pas trop affecter la probabilité de réussir le concours. En revanche, un contexte fortement concurrentiel (mesuré par le rapport entre le nombre de postes offerts au concours et le nombre de candidats) nuit à la probabilité d'être agrégé, ce qui était attendu. De même, il semble que les candidats qui ne sont pas maîtres de conférences aient une plus forte chance d'être agrégés, laissant à penser que les outsiders possèdent un avantage par rapport aux insiders.

\subsection{Une réussite plus marquée pour les publiants}

Parmi toutes les caractéristiques qui peuvent expliquer le taux de réussite au concours externe d'agrégation (cf. tableau 1), la qualité scientifique reste un critère d'importance ${ }^{24}$. Puisque nous observons le nombre de publications anglophones, francophones et le nombre de livres publiés, nous avons construit un indicateur très synthétique : le fait de n'avoir aucune publication ou d'en avoir au moins une, quel que soit son type, avant de passer le concours. En moyenne, moins de la moitié des candidats $(48,3 \%)$ possède au moins une publication recensée avant de présenter le concours. Chez les candidats qui réussissent le concours, le taux s'élève à $51,3 \%$. Il semble donc que les agrégés soient davantage des publiants ${ }^{25}$. En outre, le graphique 2 souligne que la proportion de publiants et de non-publiants, selon la réussite, varie beaucoup au fil des concours. Ainsi, lors des trois derniers concours, aucun candidat sans aucune publication n'a été agrégé. Ces statistiques éclairent donc sur l'importance de la production scientifique pour réussir le concours, voire sur son poids grandissant.

Graphique 2: Taux de réussite par concours et selon le statut de publiants ou non

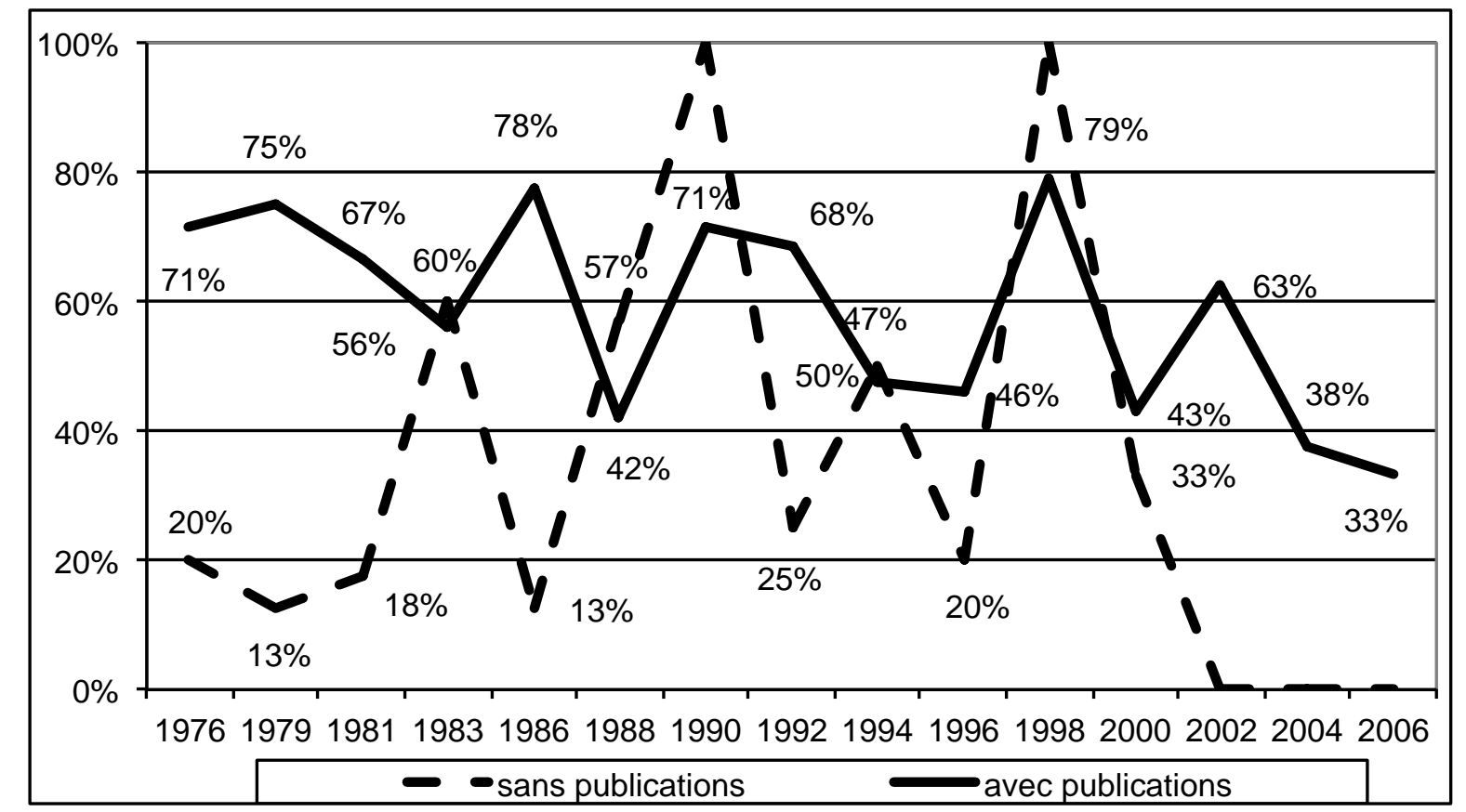

NB : statistiques données pour le sous-échantillon cylindré

\footnotetext{
${ }^{24}$ Cela est conforme aux résultats d'études précédentes : Long et al, art. cit. et Combes et al, art. cit.

${ }^{25}$ Par «publiants », nous entendons tout simplement les enseignants-chercheurs pour lesquels des publications ont été recensées selon les catégories choisies au départ. Aucune dimension qualitative n'est sous-entendue ici.
} 


\subsection{La présence du directeur de thèse dans le jury : le déterminant essentiel}

Pourtant, la qualité scientifique ne semble pas être le seul facteur de réussite, la mobilisation du réseau du candidat semblant être autant, voire plus importante, comme l'ont montré les études sur le concours d'agrégation en sciences économiques ${ }^{26}$. Nous avons donc focalisé l'attention sur une des variables de réseau : la présence du directeur de thèse comme membre du jury d'agrégation qui examine la candidature de l'individu. La fréquence observée de réussite au concours est de $83,3 \%$ pour les candidats ayant leur directeur dans le jury, contre $45,7 \%$ pour les autres. Le graphique 3 souligne que, pour sept concours sur quinze, la présence du directeur dans le jury est associée avec la réussite maximale. Notons aussi que parmi les agrégés, la part des non-publiants est de 33,3\% chez ceux ayant leur directeur dans le jury, contre $26,3 \%$ chez les autres agrégés.

Graphique 3: Taux de réussite par concours et selon la présence du directeur de thèse dans le jury

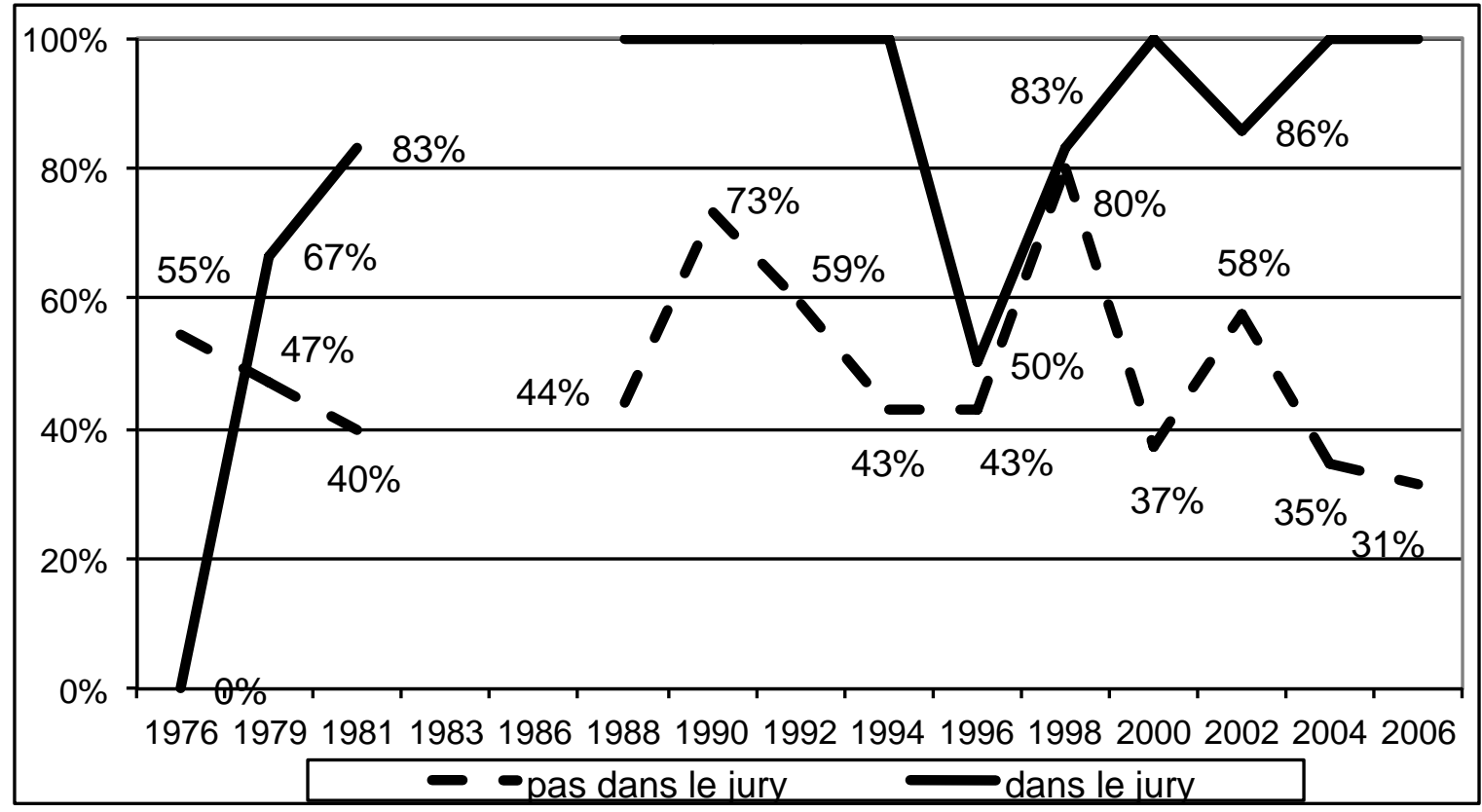

NB : statistiques données pour le sous-échantillon cylindré

Il apparaît donc clairement que la présence du directeur de thèse dans le jury pèse d'un poids essentiel, susceptible même de compenser l'insuffisance voire l'absence de publications.

Ces statistiques descriptives informent donc sur les déterminants de la réussite au concours d'agrégation en sciences de gestion. Pourtant, elles ne suffisent pas, car elles ne proposent qu'une analyse univariée du phénomène. Or, les déterminants de la réussite sont multiples et il est nécessaire d'utiliser une méthode qui assure une analyse toutes choses égales par ailleurs pour établir l'effet réel de chaque facteur explicatif. Nous recourons donc à une méthodologie économétrique, en estimant par des modèles probit la probabilité de réussir le concours.

\footnotetext{
${ }^{26}$ Voir Combes et al., art. cit.
} 


\section{Une analyse multivariée de la réussite au concours}

Ce paragraphe présente les résultats obtenus à l'issue de l'analyse économétrique. Il s'agit essentiellement d'estimer les probabilités de la réussite au concours, toutes choses égales par ailleurs, selon quelques variables déterminantes.

\subsection{L'estimation de la probabilité de réussir le concours d'agrégation}

Nous estimons les déterminants de la réussite qui se mesure par une variable binaire (être agrégé ou pas). Nous utilisons donc un modèle probit en introduisant une repondération pour redresser l'échantillon de travail et le rendre équivalent à la population. Nous proposons trois estimations : l'une pour l'ensemble de l'échantillon et deux autres sur des sous-échantillons définis selon la date du concours, c'est-à-dire avant et après 1996. Cette année charnière a été choisie car elle correspond au début de l'essor du nombre de postes ouverts en sciences de gestion au grade de maître de conférences. Elle permet aussi d'avoir des sous-échantillons équilibrés. Les estimations sur des sous-échantillons permettent de tester si les déterminants de la réussite ont évolué au fil du temps. Les résultats de cette estimation sont présentés dans le tableau 2.

Avant de les commenter en détail, discutons de la qualité des estimations. En dépit de facteurs explicatifs de la réussite au concours qui n'ont pas pu être observés (qualité des laboratoires, conditions de travail, caractéristiques personnelles et familiales, etc.), nos estimations aboutissent à plus de $85 \%$ de prédictions correctes pour l'ensemble de l'échantillon. Sachant qu'une prédiction naïve aboutirait à un score de $50 \%$, nos estimations fournissent donc une large part d'explications du phénomène étudié. Elles apparaissent donc particulièrement robustes. Elles nous permettent de mettre en évidence deux séries de facteurs explicatifs de la réussite au concours : des facteurs tels que l'âge ou le parcours antérieur des candidats et des facteurs relevant des publications et du réseau. 
$\underline{\text { Tableau 2: Déterminants de la réussite au concours externe }}$

\begin{tabular}{|c|c|c|c|c|c|c|}
\hline & & & \multirow{2}{*}{\multicolumn{2}{|c|}{ Avant 1996}} & \multirow{2}{*}{\multicolumn{2}{|c|}{ Après 1996}} \\
\hline & \multicolumn{2}{|c|}{ Tous } & & & & \\
\hline & $\begin{array}{c}\text { Effet } \\
\text { marginal }\end{array}$ & $\begin{array}{c}\text { T de } \\
\text { Student }\end{array}$ & $\begin{array}{c}\text { Effet } \\
\text { marginal }\end{array}$ & $\begin{array}{c}\text { T de } \\
\text { Student }\end{array}$ & $\begin{array}{c}\text { Effet } \\
\text { marginal }\end{array}$ & $\begin{array}{c}\text { T de } \\
\text { Student }\end{array}$ \\
\hline fem : être de sexe féminin & $-0,069$ & $-0,72$ & $-0,191$ & $-1,29$ & 0,084 & 0,65 \\
\hline these_concours: temps entre la thèse et la candidature au concours & $-0,057$ & $-4,48^{\star \star \star}$ & $-0,034$ & $-3,13^{* * *}$ & $-0,072$ & $-3,24^{* \star \star}$ \\
\hline age_these : age lors de la thèse & $-0,031$ & $-1,98^{\star *}$ & $-0,011$ & $-1,92^{* *}$ & $-0,031$ & $-2,30^{\star *}$ \\
\hline nb_tent : nombre de tentative & $-0,105$ & $-1,43$ & $-0,181$ & $-1,38$ & $-0,065$ & $-0,94$ \\
\hline poste_candidat : degré de concurrence & 0,919 & $3,75^{* * *}$ & 0,904 & $2,65^{\star \star *}$ & 1,596 & $3,46^{* \star *}$ \\
\hline compta_fi : avoir une thèse en comptabilité ou finance & 0,310 & $2,86^{* * *}$ & 0,288 & $1,73^{*}$ & 0,262 & $1,81^{*}$ \\
\hline marketing : avoir une thèse en marketing & 0,448 & $4,24^{* * *}$ & 0,512 & $3,32^{* \star *}$ & 0,262 & 1,60 \\
\hline grh : avoir une thèse en GRH & 0,311 & 1,59 & 0,394 & 1,56 & 0,253 & 0,96 \\
\hline logistiq : avoir une thèse en logistique & 0,190 & 1,08 & 0,207 & 0,75 & 0,078 & 0,39 \\
\hline si : avoir une thèse en comptabilité ou finance & 0,266 & 1,31 & 0,270 & 0,88 & 0,162 & 0,46 \\
\hline autre_disc : avoir une thèse en dehors des sciences de gestion & $-0,087$ & $-1,85^{\star}$ & $-0,021$ & $-1,97^{* *}$ & 0,098 & $1,80^{*}$ \\
\hline strat : avoir une thèse en management stratégique & ref. & & ref. & & ref. & \\
\hline mcf : être maître de conférences au moment du concours & $-0,082$ & $-2,16^{* *}$ & $-0,112$ & $-1,95^{\star}$ & 0,066 & 1,36 \\
\hline pub_angl : nombre de publications dans des revues anglophones & 0,055 & $2,30^{\star *}$ & 0,007 & $1,81^{*}$ & 0,071 & $2,23^{\star *}$ \\
\hline pub_fr: nombre de publications dans des revues francophones & 0,039 & $3,85^{\star * *}$ & 0,032 & $2,46^{\star *}$ & 0,012 & $3,99^{\star \star *}$ \\
\hline livre: nombre de livres publiés & 0,027 & $2,33^{\star *}$ & 0,027 & $2,56^{\star \star *}$ & 0,000 & $-0,02$ \\
\hline directeur_jury : avoir son directeur de thèse dans le jury & 0,368 & $2,54^{\star *}$ & 0,343 & $1,66^{*}$ & 0,553 & $2,63^{\star * *}$ \\
\hline direct_autrejury : avoir son directeur de thèse membre d'un autre jury & 0,083 & 0,97 & 0,110 & 0,82 & 0,063 & 0,50 \\
\hline coauteur_jury : avoir un coauteur dans un jury & 0,053 & $2,16^{\star *}$ & 0,132 & $3,15^{\star \star \star}$ & 0,282 & $2,22^{* *}$ \\
\hline Nombre d'observations & & & 2 & & & \\
\hline Log de vraisemblance & &, 48 & -95 & & & \\
\hline prédictior & & $0 \%$ & 77.5 & & & \\
\hline
\end{tabular}

NB : Une correction de l'hétéroscédasticité a été réalisée suivant la méthode de White (1982)

L'échantillon a été redressé de manière à tenir compte des informations manquantes.

${ }^{* * *}$ : significatif à $1 \% ;{ }^{* *}$ : significatif à $5 \%,{ }^{*}$ : significatif à $10 \%$ 


\subsection{Le rôle des caractéristiques personnelles}

Parmi les différents facteurs de réussite étudiés figurent tout d'abord les caractéristiques du candidat et son parcours antérieur. Contrairement aux études américaines, nous ne mettons en évidence aucun effet de genre : les femmes ( $\mathrm{fem}$ ) ont, toutes choses égales par ailleurs, la même probabilité de réussite que les hommes. Pourtant, ce résultat ne doit pas être interprété comme une parfaite égalité entre hommes et femmes lors du concours, puisqu'on observe une large sous-représentation des femmes candidates par rapport à leurs homologues masculins. $20 \%$ des candidats sont des femmes alors que ces dernières représentent par exemple plus de $40 \%$ des maîtres de conférences en sciences de gestion en 2006. Les femmes postulent donc moins au concours d'agrégation, mais une fois qu'elles ont postulé, elles ont les mêmes chances de réussite. Il reste donc à comprendre les raisons pour lesquelles elles postulent moins souvent que les hommes. Les résultats d'une enquête précédente sur les carrières des femmes à l'Université ont mis en évidence la complexité et la variété des facteurs explicatifs qui combinent à la fois des mécanismes organisationnels et des attitudes individuelles ${ }^{27}$.

Nos résultats économétriques confirment également l'existence d'une prime à la jeunesse, puisque être jeune lors de sa soutenance (age_these) et passer ensuite rapidement le concours d'agrégation (these_concours) constituent deux variables qui agissent significativement et positivement sur la probabilité de réussir. Cet effet semble même se renforcer pour les concours organisés après 1996. En outre, comme le laissaient penser les statistiques descriptives, le fait d'occuper un poste de maître de conférences $(m c f)$, et être normalement considéré comme un insider mieux informé, ne s'avère pas forcément un avantage pour être agrégé. Au contraire, ne pas être enseignant-chercheur (mais par exemple chercheur CNRS, personne issue du monde professionnel,...) accroît de $8 \%$ les chances de réussir par rapport aux maîtres de conférences.

Cependant, nous mettons en évidence que le statut du candidat avant le concours a un effet complètement opposé selon les cohortes. Pour les concours d'avant 1996, le fait d'être maître de conférences baisse de $17 \%$ la probabilité d'être agrégé, alors qu'après 1996, ce statut n'affecte plus de manière significative la probabilité de réussite : les chances de réussite sont donc les mêmes, quel que soit le statut au moment du concours, à partir de 1996. L'avantage marginal des « outsiders » a donc tendance à disparaître au fil du temps. Ce résultat peut être interprété comme caractérisant la transformation progressive du concours d'agrégation en un véritable dispositif de gestion de carrière au lieu d'être un concours de recrutement, au sens premier du terme. Autrement dit, la carrière organisationnelle des maîtres de conférences en sciences de gestion passe, de plus en plus souvent au fil des cohortes et désormais très majoritairement (voir données infra), par la réussite au concours d'agrégation externe.

Concernant les spécialités de thèse, on constate surtout une différence entre les candidats titulaires d'une thèse en gestion, leur spécialité important peu, et les candidats ayant soutenu leur thèse dans une autre discipline. Si, pour les concours antérieurs à 1996, cette différence n'est pas très importante, elle le devient après 1996, les docteurs en gestion montrant ensuite un taux de réussite supérieur (d'environ $10 \%$ ). Ce résultat peut aussi tenir au fait que la discipline des sciences de gestion ayant été constituée à la fin des années 70, lors des premiers concours, peu de candidats pouvaient se prévaloir d'un doctorat de gestion, la plupart possédant des doctorats d'autres disciplines, notamment d'économie ou de droit. Au fur et à

\footnotetext{
${ }^{27}$ Pour une analyse plus détaillée, on pourra se reporter à l'étude réalisée par Carrère (Myriam), Louvel (Séverine), Mangematin (Vicent), Musselin (Christine), Pigeyre (Frédérique), Sabatier (Mareva), Valette (Annick), Les carrières des chercheurs de l'INRA. Analyses et interprétations, Rapport de recherche, Paris et Grenoble, 2006.
} 
mesure que la discipline s'installe, des docteurs plus nombreux en gestion sont formés et deviennent alors les candidats naturels du concours d'agrégation en sciences de gestion.

Nous montrons aussi que le fait d'avoir déjà passé le concours (et échoué) n'a pas d'effet significatif sur la probabilité d'être agrégé par la suite. En outre, de manière assez attendue, un contexte peu concurrentiel (poste_candidat) favorise la réussite. Notons que ce résultat n'est pas affecté lorsque le nombre de postes réellement pourvus est pris comme référence au lieu du nombre de postes ouverts lors du concours.

\subsection{Les facteurs liés aux publications et au réseau}

Cette analyse présente l'intérêt de voir comment interviennent non seulement les facteurs de qualité scientifique mais aussi ceux liés au réseau, c'est-à-dire essentiellement le fait d'avoir son directeur de thèse ou un co-auteur dans le jury.

Nos résultats confirment d'abord que les publiants ont davantage de chances d'être agrégés. Il semble aussi se dégager une certaine hiérarchie dans les publications, mais elle est différente selon les cohortes. Avant 1996, les publications francophones ainsi que les livres sont davantage valorisés, mais cela témoigne simplement du très faible taux de publications à l'international à cette époque. Après 1996, les publications anglophones ont plus d'influence que les publications francophones, les livres n'ayant plus d'effet, ceci illustrant l'évolution de l'évaluation de la production scientifique au fil des concours.

Cependant, si de bonnes publications assurent de plus fortes chances de réussite, certaines variables de réseau ont un effet plus important que les publications. Si la présence d'un coauteur dans le jury (coauteur_jury) favorise la réussite (en augmentant la probabilité d'être agrégé d'environ 5,3\%), c'est surtout la présence du directeur de thèse qui compte. D'un point de vue quantitatif, avoir son directeur dans son jury (directeur_jury) augmente de presque $37 \%$ la probabilité de réussir le concours et même de 55\% pour la cohorte la plus récente, toutes choses égales par ailleurs.

Devant l'ampleur de cet effet, nous avons simulé pour l'échantillon complet, combien un candidat qui n'a pas son directeur dans le jury devrait avoir publié d'articles ou de livres en plus pour obtenir la même chance de réussite et ainsi compenser l'absence de soutien dans le jury. Nos simulations aboutissent au résultat suivant : un candidat qui n'a pas son directeur de thèse dans le jury doit, pour avoir une chance équivalente de réussite, avoir publié en moyenne, presque 7 articles de plus dans des revues anglophones, presque 10 articles dans des revues francophones ou plus de 13 livres en sus. L'impact du réseau apparaît donc bien supérieur à celui des publications et les ordres de grandeur sont très proches de ceux trouvés pour le concours d'agrégation en économi ${ }^{28}$. Notons en complément que la présence du directeur dans un autre jury (direct_autrejury) n'a pas d'impact significatif sur la réussite au concours. Les potentiels effets de réputation ou simplement d'information ne semblent donc pas jouer de façon significative.

\subsection{L'agrégation, un concours « vertueux »?}

Notre analyse permet de mieux comprendre les facteurs explicatifs de la réussite au concours d'agrégation externe en sciences de gestion. Ils permettent même d'ébaucher le "portraittype » du lauréat du concours d'agrégation : c'est un candidat jeune, homme ou femme, qui a soutenu sa thèse en sciences de gestion peu de temps avant le concours, qui a publié dans des

\footnotetext{
${ }^{28}$ Voir Combes et al., art cit.
} 
revues francophones et anglophones et dont le directeur de thèse est membre du jury. Sur le fond, ce portrait-type mériterait d'être affiné (des entretiens biographiques permettraient d'ouvrir des pistes intéressantes, notamment quant à l'importance des environnements organisationnels et personnels des candidats), d'autant qu'il se base uniquement sur ce qui s'est passé jusqu'à présent.

Nos résultats corroborent ainsi la plupart des discours : le concours d'agrégation accorde une grande importance aux publications. De ce point de vue, on pourrait dire qu'il remplit la mission qui lui est attribuée, consistant à identifier les meilleurs publiants qui «méritent» donc d'accéder au grade de Professeur. La logique méritocratique, l'un des fondements des sociétés démocratiques, fonctionne ainsi sans ambigüité ${ }^{29}$.

Cependant, nos résultats montrent aussi que le facteur «publications » n'est pas le facteur dominant: c'est le fait d'avoir son directeur de thèse dans le jury qui apparait comme essentiel. Cette réalité amène à s'interroger sur le caractère prétendument égalitaire et équitable du concours, pourtant considéré comme la vertu essentielle et la justification de tout concours. En outre, cela soulève une question importante en matière de justice organisationnelle selon ses deux dimensions de justice distributive et de justice procédurale ${ }^{30}$. $\mathrm{Du}$ point de vue de la justice distributive, le concours, dont nous avons décrit le fonctionnement réel, risque d'être mal perçu, voire remis en cause : en quoi un tel concours permet-il de réellement reconnaître la qualité du travail de recherche, évalué à l'aune des publications des candidats, si ces dernières passent au second plan ? Du point de vue de la justice procédurale, c'est le sentiment de justice ressenti à l'égard de ce mode de promotion ce concours en particulier- qui risque d'être mis à mal : est-il normal, légitime, que les décisions de promotion au grade de professeur s'appuient sur un concours qui ne reconnaît pas en priorité le résultats des efforts et des mérites consentis, à savoir les publications?

\section{Conclusion}

Cet article proposait, à travers une étude appliquée au cas du concours d'agrégation du supérieur en sciences de gestion, d'analyser les vertus théoriques des procédures de concours, procédures censées le mieux reconnaître et sélectionner les plus méritants. Exploitant une base de données inédite, nous mettons en évidence deux résultats importants. Premièrement, le concours d'agrégation en gestion reconnaît le mérite puisque les candidats qui publient le plus ont davantage de chances de réussite. Deuxièmement, le concours ne semble pourtant pas parfaitement équitable, puisque si les chances de réussite augmentent avec les publications, elles sont comparativement nettement plus élevées quand le candidat a son directeur de thèse dans le jury. Le concours, tel qu'il a lieu, reconnaît donc le mérite mais ne s'affranchit pas des effets de réseau. Nos résultats soulignent donc que l'agrégation est plutôt éloignée du modèle emblématique du concours de la Fonction Publique et que la situation réelle est très proche de celle d'un recrutement classique, conforme au modèle du marché, par ailleurs fort bien

\footnotetext{
${ }^{29}$ Notons cependant que sur cette base, seuls les mérites prouvés dans le domaine de la recherche sont pris en compte, et nullement les autres compétences des enseignants-chercheurs, en particulier sur la dimension pédagogique. Mais notre propos ne concerne pas directement la pertinence du concours tel qu'il est actuellement organisé.

${ }^{30}$ Cadin (Loïc) et Guérin (Francis), «Les outils d'appréciation des salariés », in Allouche (José) (coord.), Encyclopédie de gestion des ressources humaines, Paris, Vuibert, 2006, p. 26-34.
} 
analysé par la littérature ${ }^{31}$. Reste qu'aucune étude n'a été menée pour comparer les procédures de recrutement des professeurs (décentralisées ou par concours) et il est aujourd'hui encore impossible de déterminer quelle procédure est la plus efficace, à défaut d'être parfaite.

Envisagés dans le contexte actuel de débat public et de controverses quant à l'évolution des carrières des enseignants-chercheurs, nos résultats pourraient aussi permettre d'engager une réflexion approfondie sur la manière dont sont recrutés les professeurs d'université. Alors que la situation démographique du corps des professeurs en sciences de gestion devrait conduire à son renouvellement significatif, l'accès à ce corps reste encore extrêmement limité. De plus, la concurrence, aussi féroce que récente des écoles de commerce, conduit les jeunes docteurs, dont le nombre diminue, à s'éloigner de l'Université, que de nombreux rapports accusent de faible attractivité (voir par exemple le rapport de l'Académie des sciences remis à la Ministre de l'Enseignement Supérieur et de la Recherche en juillet 2008).

Plus largement, au sein même de la profession des enseignants-chercheurs en gestion, il s'agirait de repenser, dans le cadre d'universités rendues plus autonomes, la gestion des carrières, à court et moyen terme, ce qui suppose également de repenser la pertinence de ce type de concours.

\footnotetext{
${ }^{31}$ Voir notamment Eymard-Duvernay (François) et Marchal (Emmanuelle), Façons de recruter. Le jugement des compétences sur le marché du travail, Paris, Métailié, 1997
} 
ANNEXE 1: Description des variables

\begin{tabular}{|c|c|c|c|c|}
\hline Nom & Explication & Source & $\begin{array}{c}\text { Nombre } \\
\text { d'observations }\end{array}$ & $\begin{array}{c}\text { Fréquence / } \\
\text { Moyenne }\end{array}$ \\
\hline agrégés & $\begin{array}{l}=1 \text { si le candidat réussit le } \\
\text { concours }\end{array}$ & Ministère & 626 & 0,45 \\
\hline poste_candidat & $\begin{array}{l}=\text { nombre de poste } / \text { nombre de } \\
\text { candidats }\end{array}$ & Ministère & 626 & 0,40 \\
\hline nb_tentative & $\begin{array}{l}\text { = nombre de tentatives } \\
\text { précédentes }\end{array}$ & Ministère & 626 & 1,40 \\
\hline hom & $=1 \mathrm{si}$ le candidat est un homme & Ministère & 626 & 0,80 \\
\hline age_these & $=$ âge au moment de la thèse & Ministère + SUDOC & 605 & 31,37 \\
\hline $\begin{array}{l}\text { these_concour } \\
\mathrm{s}\end{array}$ & $\begin{array}{l}\text { = nombre d'années entre thèse } \\
\text { et présentation du concours }\end{array}$ & Ministère + SUDOC & 596 & 6,29 \\
\hline \multicolumn{5}{|c|}{ Publications mesurées avant le passage du concours } \\
\hline pub_fr & $\begin{array}{l}=\text { nombre de publications } \\
\text { francophones }\end{array}$ & $\begin{array}{l}\text { Bases de données } \\
\text { bibliographiques + } \\
\text { sommaires }\end{array}$ & 626 & 2,18 \\
\hline pub_angl & $\begin{array}{l}=\text { nombre de publications } \\
\text { anglophones }\end{array}$ & \begin{tabular}{|l|} 
Bases de données \\
bibliographiques
\end{tabular} & 626 & 0,47 \\
\hline livre & $=$ nombre de livres publiés & $\begin{array}{l}\text { Bases de données } \\
\text { bibliographiques }\end{array}$ & 626 & 0,99 \\
\hline \multicolumn{5}{|c|}{ Carrière dans l'enseignement supérieur } \\
\hline mcf & $\begin{array}{l}=1 \text { si le candidat est MCF au } \\
\text { moment du concours }\end{array}$ & Ministère & 626 & \\
\hline \multicolumn{5}{|c|}{ Spécialité de thèse } \\
\hline autre_spe & $\begin{array}{l}=1 \text { si le candidat n'a pas fait sa } \\
\text { thèse en gestion }\end{array}$ & SUDOC & 605 & 0,29 \\
\hline manag_strat & $\begin{array}{l}\text { = } 1 \text { si le candidat a fait sa thèse } \\
\text { en management ou stratégie }\end{array}$ & SUDOC & 605 & 0,22 \\
\hline compta_fin & $\begin{array}{l}\text { = } 1 \text { si le candidat a fait sa thèse } \\
\text { en comptabilité ou finance }\end{array}$ & SUDOC & 605 & 0,25 \\
\hline prod_logist & $\begin{array}{l}\text { = } 1 \text { si le candidat a fait sa thèse } \\
\text { en production ou logistique }\end{array}$ & SUDOC & 605 & 0,04 \\
\hline si & $\begin{array}{l}=1 \text { si le candidat a fait sa thèse } \\
\text { en système d'information }\end{array}$ & SUDOC & 605 & 0,02 \\
\hline grh & $\begin{array}{l}=1 \text { si le candidat a fait sa thèse } \\
\text { en GRH }\end{array}$ & SUDOC & 605 & 0,08 \\
\hline marketing & $\begin{array}{l}\text { = } 1 \text { si le candidat a fait sa thèse } \\
\text { en marketing }\end{array}$ & SUDOC & 605 & 0,10 \\
\hline \multicolumn{5}{|l|}{ Réseau } \\
\hline $\begin{array}{l}\text { direct_autre } \\
\text { jury }\end{array}$ & $\begin{array}{l}\text { = } 1 \text { si le directeur de thèse du } \\
\text { candidat a été membre d'un } \\
\text { autre jury }\end{array}$ & Ministère + SUDOC & 589 & 0,15 \\
\hline coauteur_jury & $\begin{array}{l}=1 \text { si un coauteur est membre } \\
\text { du jury }\end{array}$ & Ministère + SUDOC & 626 & 0,04 \\
\hline directeur_jury & $\begin{array}{l}=1 \text { si le directeur de thèse de } \\
\text { l'individu est dans le jury }\end{array}$ & Ministère + SUDOC & 548 & 0,07 \\
\hline
\end{tabular}

